\title{
Tragedy in a heartbeat: malfunctioning desmin causes skeletal and cardiac muscle disease
}

\author{
Lev G. Goldfarb¹ and Marinos C. Dalakas²
}

${ }^{1}$ National Institute of Neurological Disorders and Stroke, NIH, Bethesda, Maryland, USA. ${ }^{2}$ mmperial College London, Hammersmith Hospital Campus, London, United Kingdom.

\begin{abstract}
Muscle fiber deterioration resulting in progressive skeletal muscle weakness, heart failure, and respiratory distress occurs in more than 20 inherited myopathies. As discussed in this Review, one of the newly identified myopathies is desminopathy, a disease caused by dysfunctional mutations in desmin, a type III intermediate filament protein, or $\alpha B$-crystallin, a chaperone for desmin. The range of clinical manifestations in patients with desminopathy is wide and may overlap with those observed in individuals with other myopathies. Awareness of this disease needs to be heightened, diagnostic criteria reliably outlined, and molecular testing readily available; this would ensure prevention of sudden death from cardiac arrhythmias and other complications.
\end{abstract}

\section{Background}

Desminopathy was originally described as skeletal and cardiac myopathy characterized by bilateral weakness in distal leg muscles spreading proximally and leading eventually to tetraparesis and wheelchair dependence (1). Weakness sometimes involves trunk, neck flexors, and facial muscles. In disease variants marked with early onset cardiomyopathy, patients experience dizziness and syncopal and fainting episodes associated with conduction blocks and require insertion of a permanent pacemaker $(1,2)$. Respiratory muscle weakness is a frequent component of desminopathy.

Desminopathy belongs to a genetically heterogeneous group of disorders named myofibrillar myopathies, which are caused by mutations in the following genes: desmin $(D E S), \alpha B$-crystallin $(C R Y A B)$, myotilin (MYOT), Z band alternatively spliced PDZ-containing protein (ZASP), filamin C (FLNC), or Bcl-2-associated athanogene-3 (BAG3) $(2,3)$. The common pathological pattern in myofibrillar myopathy is dissolution of myofibrils, aggregation of degraded myofibrillar products, and ectopic expression of proteins (2), but clinical manifestations are remarkably different. In this Review we discuss myofibrillar myopathies associated with mutations in DES $(4,5)$ and CRYAB (6).

Desmin is the main intermediate filament (IF) protein expressed in cardiac, skeletal, and smooth muscle. It interacts with other proteins to form a continuous cytoskeletal network that maintains a spatial relationship between the contractile apparatus and other structural elements of the cell, thus providing maintenance of cellular integrity, force transmission, and mechanochemical signaling (Figure 1). Desmin is much more abundant in heart muscle ( $2 \%$ of total protein) than in skeletal muscle $(0.35 \%)$ and is a major component of Purkinje fibers, the specialized myocardial conduction system that enables the heart to contract in a coordinated fashion (7). $\alpha$ B-crystallin serves as a chaperone for desmin and other proteins, preventing them from aggregating under various forms of stress (8).

\section{Desmin mutations and their structural consequences}

Human desmin is encoded by a single-copy gene (DES) located on chromosome $2 \mathrm{q} 35$ (9). DES encompasses nine exons within an

Conflict of interest: The authors have declared that no conflict of interest exists. Nonstandard abbreviations used: $C R Y A B, \alpha \mathrm{B}$-crystallin; $D E S$, desmin; IF, intermediate filament.

Citation for this article: J. Clin. Invest. 119:1806-1813 (2009). doi:10.1172/JCI38027. 8.4-kb region and encodes 470 amino acids (10). The gene is highly conserved among vertebrate species. In accordance with its function, desmin is organized into three domains - a highly conserved $\alpha$-helical core of 308 amino acid residues flanked by globular $\mathrm{N}$ - and C-terminal structures (known as the "head" and "tail" domains, respectively) (11). The $\alpha$-helical core maintains a sevenresidue (heptad) repeat pattern that guides two polypeptides to form a homopolymeric coiled-coil dimer, the elementary unit of the desmin IF; the heptad periodicity within the helical rod is interrupted in several places, resulting in four consecutive helical segments (known as 1A, 1B, 2A, and 2B) connected by short, nonhelical linkers (12).

Thus far, the number of known disease-causing DES mutations has reached 45 (Figure 2): 40 are missense mutations, 3 are small in-frame deletions of between one and seven amino acids, 1 is an exon-skipping mutation, and 1 is an insertion of a single nucleotide resulting in premature translation termination. Thirteen of the mutations are recurrent, appearing in more than one unrelated family. Five mutations are in the head domain, one is in the $1 \mathrm{~A}$ helical segment, five are in the $1 \mathrm{~B}$ segment, 23 reside in the $2 \mathrm{~B}$ helical segment, and 11 are in the tail domain. No mutations have thus far been identified in the 2A helical segment.

The exon-skipping mutation is caused by a series of alterations in the highly conserved intronic donor and acceptor splice sites flanking exon 3 and results in exon 3 deletion (13). Binding sites to nebulin (14) and perhaps other interacting proteins are encoded within this exon. The heterozygous single-nucleotide (adenine) insertion mutation occurs at the third position of codon 241 and results in a frameshift leading to serial amino acid replacements and eventually a premature termination signal at codon 245; this mutation is predicted to create a truncated desmin molecule with a molecular weight of $27 \mathrm{kDa}(15)$.

Analysis of the $2 \mathrm{~B}$ helical segment has attracted maximal attention because most of the known DES mutations occur in the portion of the gene that encodes this region. Eleven of the 23 missense mutations found in the $2 \mathrm{~B}$ helical segment introduce proline. Proline is not normally present in the desmin helical rod and is known to be a potent helix breaker; its dihedral angle is fixed at $-65^{\circ}$, creating a kink in the protein structure (16). In addition, proline destabilizes $\alpha$-helices by its inability to form hydrogen bonds. In mutagenesis experiments, the introduction of proline residues 


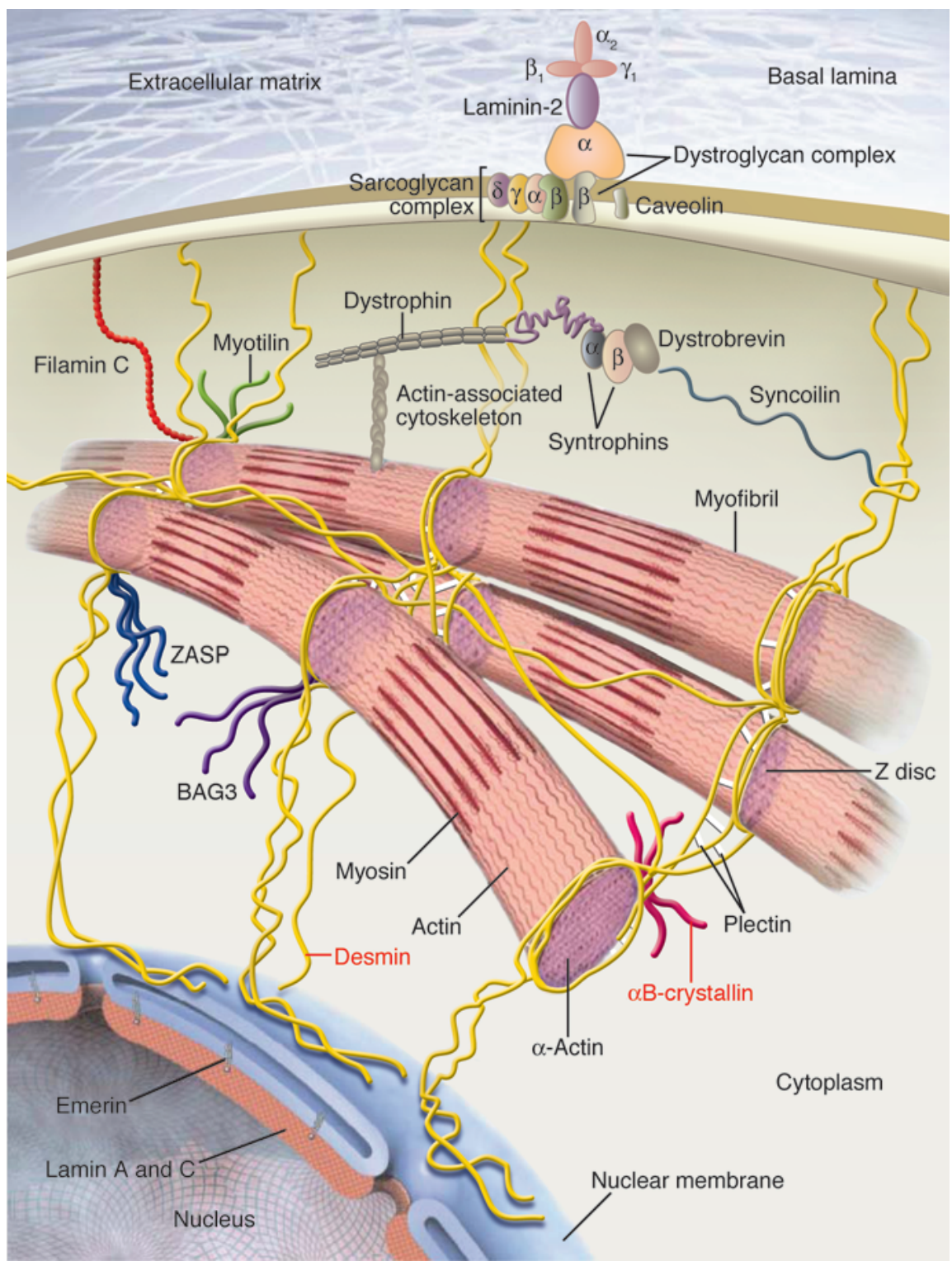

\section{Figure 1}

Molecular cytoarchitecture of a myocyte, featuring proteins involved in skeletal and cardiac myopathies. Desmin is the main muscle IF protein. It interacts with other proteins to support myofibrils at the level of the $Z$ disc and forms a continuous cytoskeletal IF network that maintains a spatial relationship between the contractile apparatus and other structural elements of the cell. Desmin provides maintenance of cellular integrity, force transmission, and mechanochemical signaling. Mutations in other sarcomeric and cytoskeletal proteins (plectin, myotilin, filamin $\mathrm{C}, \alpha \mathrm{B}$-crystallin, $\mathrm{Z}$ band alternatively spliced PDZ-motif protein [ZASP], and BCL2associated athanogene 3 [BAG3]) cause neuromuscular disorders. Adapted with permission from New England Journal of Medicine (61).

Another thoroughly examined desmin structural element is the YRKLLEGEESRI motif, positioned at the C-terminal end of the 2B helical segment (Figure 2). Starting with several amino acids preceding the YRKLLEGEESRI motif and through the YRKLL peptide, the coiled-coil structure loosens and the $\alpha$-helices gradually separate, eventually bending away from each other at EGEE (22). Two well-studied mutations (p.Arg406Trp and p.Glu413Lys) are located within this structure, and two other mutations reside in the preceding sequence (p.Asp399Tyr and p.Glu401Lys). These mutations damage structural and functional relationships. The p.Arg406Trp mutation replaces arginine with a larger tryptophan residue, making the helix at least a half turn longer and thereby changing the peptide structure and symmetry $(23,24)$. Filament assembly studies indicate that the p.Arg406Trp mutant has a reduced ability to support longitudinal annealing as well as radial compaction (25). The p.Asp399Tyr, p.Glu401Lys, and into desmin resulted in the generation of short, thick, and kinked abnormally assembled filaments (17).

The C-terminal part of the desmin $\alpha$-helical core domain contains a discontinuity in the heptad repeat pattern, a "stutter" (Figure 2), which is equivalent to an insertion of four extra residues at the end of the eighth heptad of the $2 \mathrm{~B}$ helical segment (18). The stutter is an obligatory feature of all IF proteins, and its position is absolutely conserved $(19,20)$. As a compensation for the stutter, the coiled coil slightly unwinds in the vicinity of the stutter. Four known mutations (p.Arg355Pro, p.Ala357Pro, p.Ala360Pro, and p.Glu359_Ser361del) occur within the stutter or in the immediate neighborhood. Molecular modeling of p.Glu359_Ser361del indicates that it creates a second stutter adjacent to the normally existing one, causing additional local unwinding of the coiled coil (21). The p.Asn366del mutation converts the stutter into a heptad repeat stammer, which leads to overwinding of the coiled coil; both mutations are thus expected to result in altered coiled-coil geometry within the $2 \mathrm{~B}$ helical segment (21).
p.Arg406Trp mutations disrupt potentially critical intrahelical and interhelical salt-bridge interactions within desmin dimers (23), and the p.Glu413Lys mutation alters electrostatic interactions that are important for proper dimer-dimer assembly (26).

As noted above, eleven disease-causing mutations are located within the 61-amino acid-residue tail domain of desmin. Although the tail lacks heptad repeats, it regulates longitudinal head-to-tail tetramer assembly and controls lateral packing, stabilization, and elongation of the higher order filament structures (27-29). Assembly studies have shown that some tail mutants form less compact filaments with highly irregular diameters and exert dominantnegative effects when mixed with wild-type desmin (30). The tail domain is also involved in interactions between desmin and other cytoskeletal proteins (29).

\section{$\alpha B$-crystallin mutations}

$\alpha \mathrm{B}$-crystallin belongs to the small heat shock protein family of chaperones, which includes hsp20, hsp22, hspB2, hspB3, hsp25, 


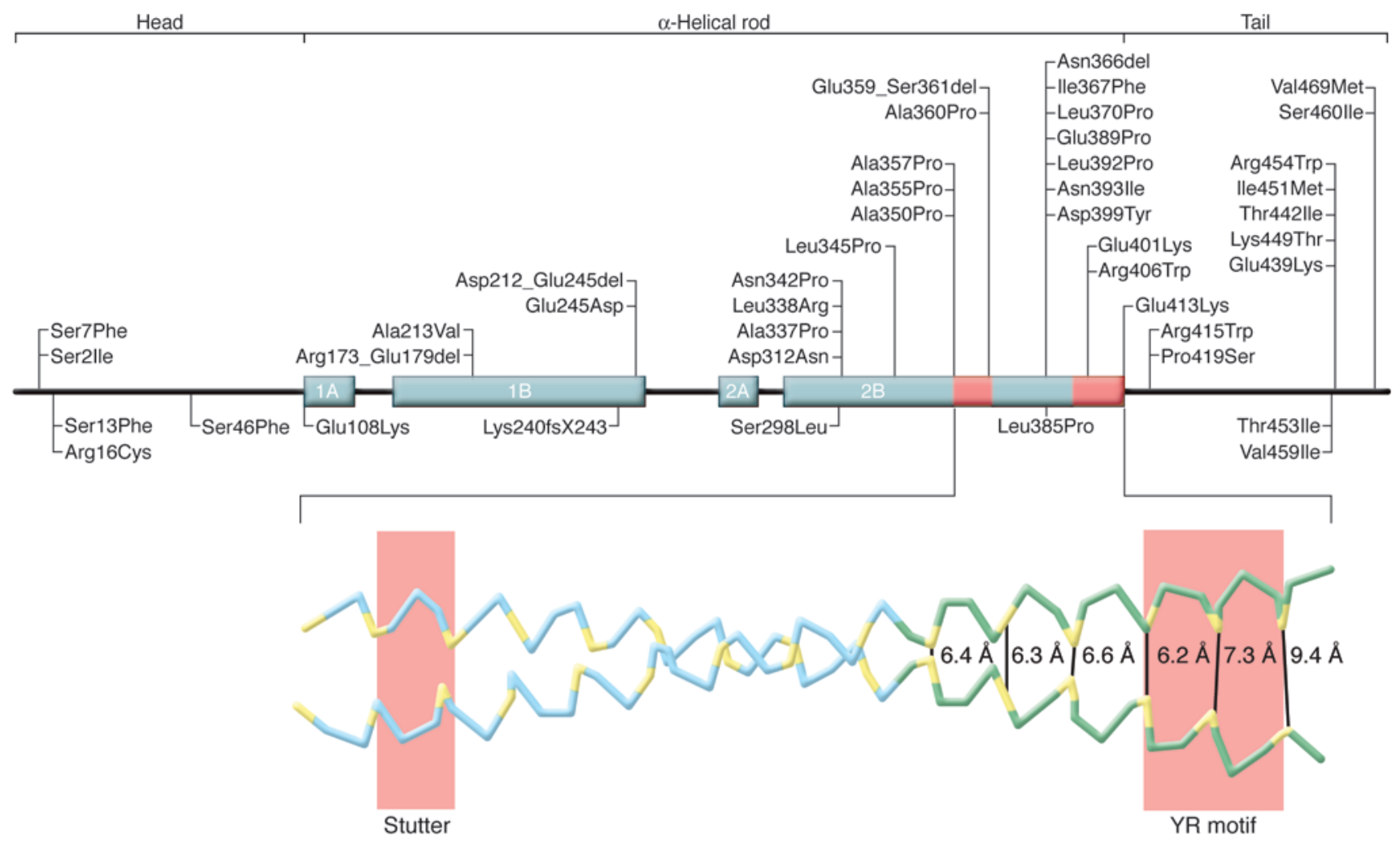

Figure 2

Updated chart of desmin mutations. Top: Desmin is composed of an $\alpha$-helical rod containing 303 amino acid residues, flanked by globular $\mathrm{N}$ - and C-terminal structures (known as the head and tail domains, respectively). The rod is interrupted in several places, resulting in four consecutive $\alpha$-helical segments, 1A, 1B, 2A, and 2B, connected by short, nonhelical linkers. Helical segment 2B contains a discontinuity in the heptad repeat pattern (known as a stutter) and an YRKLLEGEE motif (red boxes within the protein structure). Most pathogenic desmin mutations reside within either the 2B helical segment or the tail. Bottom: Molecular model of desmin coiled-coil segment based on analogy with crystallographic data for human vimentin. The stutter is a discontinuity in the heptad repeat pattern equivalent to an insertion of four extra residues, an absolutely conserved feature of all IF proteins. Starting with several amino acids preceding the YR motif and through the YR peptide, the coiled-coil structure loosens and the monomers gradually separate, eventually bending away from each other. The angles and distances between residue positions in the 2B C-terminal helical segment are shown. The bottom portion has been adapted with permission from Journal of Molecular Biology (22).

hsp27, and myotonic dystrophy kinase-binding protein. Although originally classified as a lens protein (31), $\alpha \mathrm{B}$-crystallin is abundant in cardiac and skeletal muscle (32). Chaperones respond to stressful conditions by binding to unfolded proteins, preventing their denaturation and aggregation $(8,33)$. By acting as a chaperone for desmin and cytoplasmic actin, $\alpha \mathrm{B}$-crystallin helps to maintain cytoskeletal integrity (34). $\alpha \mathrm{B}$-crystallin also participates in a number of other cellular processes, including compartment targeting, degradation, and signaling. A heterozygous CRYAB p.Arg120Gly mutation has been identified in a multigenerational French family with phenotypic characteristics of desminopathy (6, $35)$. The Arg120 residue is located in the region of $\alpha \mathrm{B}$-crystallin that is conserved among the small heat shock proteins. Structural and functional studies indicate that the mutant $\alpha \mathrm{B}$-crystallin has a diminished chaperone function $(36,37)$. Two other mutations, c.464_465CTdel and p.Gln151X, both resulting in a truncated $\alpha \mathrm{B}$-crystallin, have also been identified as the cause of a disease with phenotypic characteristics of myofibrillar myopathy (38).

\section{Molecular pathogenesis}

Identification of pathogenic mutations in desmin and $\alpha B$-crystallin, analysis of underlying disease phenotypes, and successful modeling of underlying conditions in cell cultures and transgenic mice has helped to understand critical pathogenic events in desminopathies.

Animal models. Studies of DES-knockout mice have shown that, although they develop normally and are fertile, lack of a desmin IF network prevents spatial organization of cellular components (39-42). Abnormalities include loss of the lateral alignment of myofibrils, perturbation of myofibril anchorage to the sarcolemma and nuclear matrix, loss of nuclear shape and positioning, swollen mitochondria, and unusual distribution of myosins $(40,41)$.

After birth, irregularities in myofibrillar organization are predominantly observed in weight-bearing muscles such as M. soleus (the calf) and continually and extensively used muscles such as the tongue, diaphragm, and heart $(39,40,42)$. Mitochondria increase in size and number, lose correct positioning, and eventually degenerate (41). Older animals show characteristic morphology of skeletal muscle dystrophy (40). Cardiac muscle is most susceptible to the lack of desmin; early in postnatal life, mice develop cardiomyopathy manifest by lysis of individual cardiomyocytes, invasion of macrophages, varying degrees of calcification, and finally fibrosis $(41,42)$. Disorganized, distended, and nonaligned fibers are observed in the diaphragm (41).

In transgenic mice expressing the p.Arg173_Glu179del desmin mutation that is associated with desminopathy in humans, exami- 

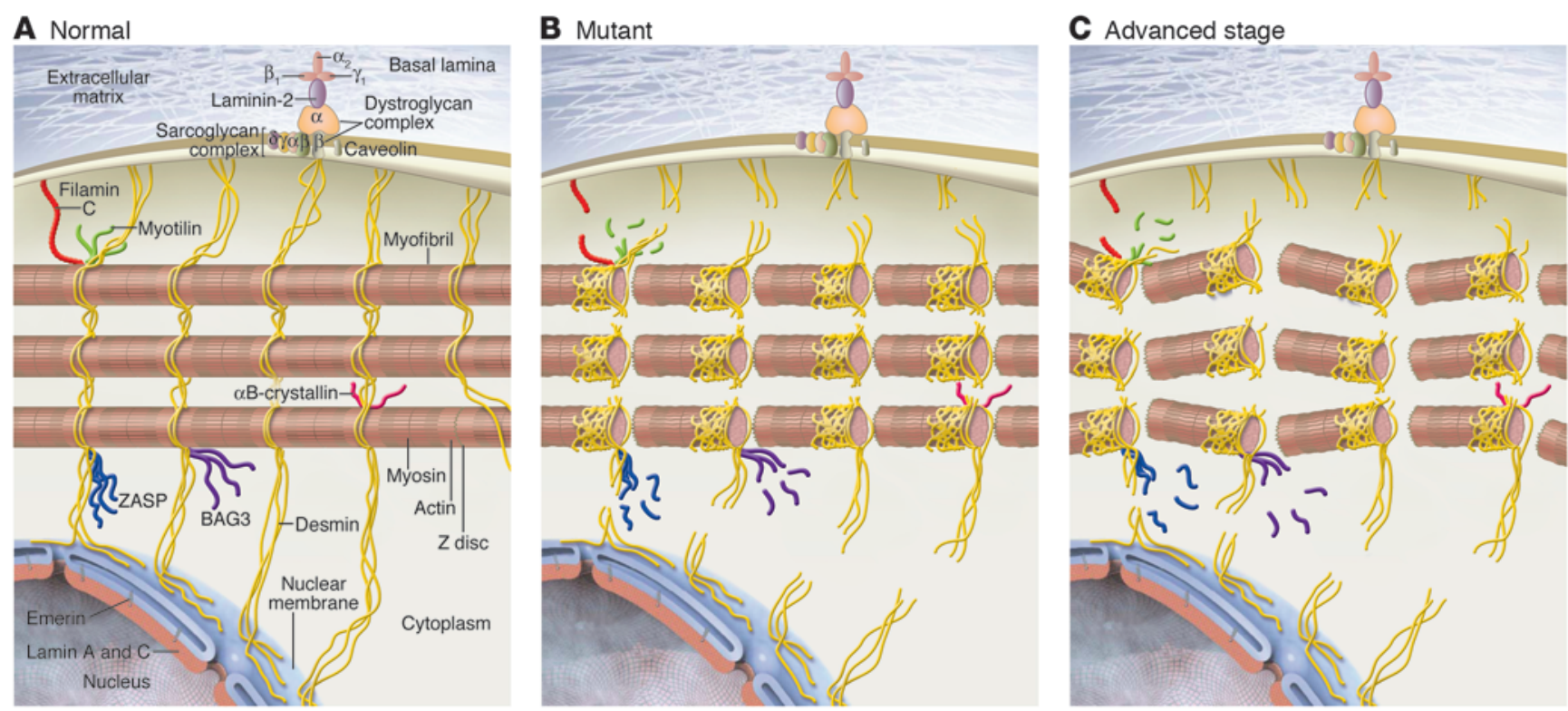

Figure 3

Schematic representation of the destructive effects of desmin mutations in muscles of patients with desminopathy. (A) Normal molecular cytoarchitecture of a myocyte (as presented in Figure 1). (B) Mutant desmin causes disorganization of the $Z$ discs and affects the integrity of the cellular IF network. (C) Fragmented desmin filaments form insoluble deposits that accumulate and eventually cause myopathy by disrupting the myofibrils, breaking the connections between them and upsetting the binding of the myofibrils to cellular membranes. Adapted with permission from New England Journal of Medicine (61).

nation of the myocardium revealed an accumulation of chimeric intracellular aggregates containing desmin and other cytoskeletal proteins (43). Such aggregates are not seen in DES-knockout mice, supporting the hypothesis that mutant misfolded desmin protein acts as a seed for the formation of protein inclusions in desminopathy. The aggregates disrupt the continuity and overall organization of the desmin network throughout the cell (43). They appear as electron-dense granulofilamentous structures proximal to the nucleus and throughout the intermyofibrillar space. In transgenic mice carrying the desminopathy-causing human p.Leu345Pro mutation, there is a striking abnormality of mitochondrial morphology and $\mathrm{Ca}^{2+}$ handling, suggesting that the mutation undermines mitochondrial integrity in muscle cells (44).

Chaperones assist normal protein folding and enhance ubiquitination and proteasomal degradation of abnormally constructed proteins; they also help to restore proteins to their native conformation after these proteins have been misfolded by heat, ischemia, chemotoxicity, or other cellular stresses (36). Expressed in SW13 and BHK21 cells, the desminopathy-associated p.Arg120Gly $\alpha B$-crystallin mutation causes the formation of abnormal intracellular aggregates that contain both desmin and $\alpha \mathrm{B}$-crystallin (6). Transgenic mice expressing this mutation show an accumulation of abnormal desmin and $\alpha \mathrm{B}$-crystallin-reactive aggregates in cardiomyocytes $(45,46)$. A chaperone assay has been used to demonstrate that the p.Arg120Gly mutant $\alpha \mathrm{B}$-crystallin is functionally deficient (47). Addition of either wild-type $\alpha B$-crystallin or HSP27 to the system prevents the formation of aggregates (37).

Filament assembly assays. Analysis of filament assembly behavior by individual desmin mutants in in vitro experiments (48) and various cell lines (49) has demonstrated that some mutants allow filament formation to take place (p.Ala213Val, p.Glu245Asp, p.Ala360Pro,
p.Glu389Pro, p.Asn393Ile, and p.Asp399Tyr) but change the number of subunits per cross-section as compared with wild-type desmin (50). Other mutants interfere with the assembly process and, according to the observed patterns, can be classified into three groups: mutations compromising longitudinal annealing properties and forming short, segmented filaments (p.Leu385Pro and p.Arg406Trp); mutants causing enhanced adhesiveness, which leads to filament aggregation (p.Ala337Pro, p.Asn342Pro, and p.Ala357Pro); and mutants that facilitate rapid disintegration of assembly precursors and form aggregates (p.Leu345Pro, p.Arg350Pro, and p.Leu370Pro) (50). Filament formation by assembly-deficient mutant desmin can be rescued in some cases by wildtype desmin (25). In many other cases, however, the mutant protein drives the wild-type protein into abnormal aggregates (30).

Cell transfection studies. The effects caused by desmin mutations have also been evaluated in two types of cells. SW13(vim-), which is a human epithelial cell line that does not express type III IF proteins, is ideal for assessing the ability of mutant desmin to produce a filamentous network by itself (17). C2C12 cells, which are mouse myoblasts, and other cells that express muscle-specific endogenous type III IF proteins allow evaluation of the effects desmin mutations may exert on a preexisting filamentous network (23). Experimentally induced (17) and most naturally occurring human desmin mutants (49) are unable to generate their own IF network and frequently disrupt the preexisting endogenous IF network (Figure 3).

Aggregate-eliminating pathways. Misfolded desmin molecules escape proteolytic breakdown and attract other cytoskeletal and ectopic proteins into high-molecular-weight insoluble chimeric aggregates (51). Overexpression of mutant desmin in cultured cardiac myocytes (52) and transgenic mice (53) inhibits the ubiquitin-proteasome system. When the degrading capacity of this 


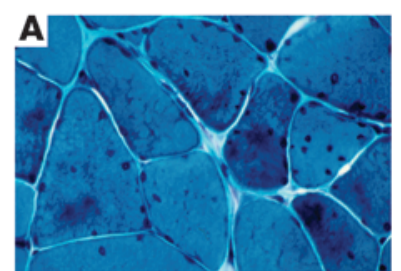

B
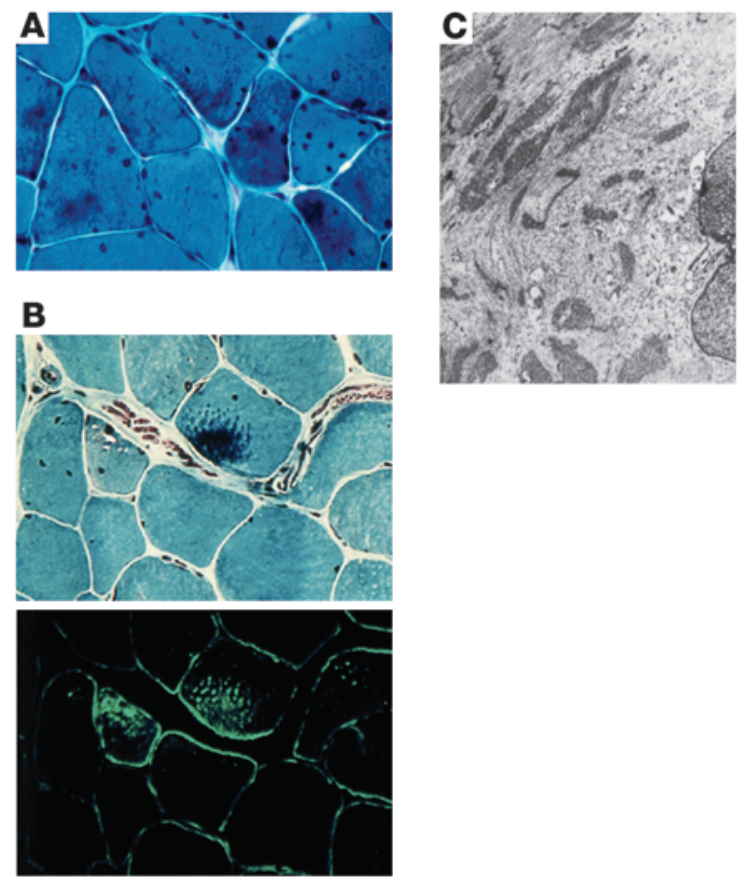

system is exceeded, misfolded proteins accumulate in perinuclear electron-dense bodies known as aggresomes (37). The aggresomes identified in cardiomyocytes of a mouse expressing the CRYAB p.Arg120Gly mutation are similar to the accumulations of primary toxic oligomers in neurons of patients with Alzheimer disease (54) and myocytes of patients with inclusion body myositis $(55,56)$. Analysis of aggresome composition also suggests that they contain an amyloid oligomer representing a primary toxic species (54).

Toxic aggregates trigger autophagy as a mechanism for clearing the aberrant proteins from the muscle fiber $(56,57)$. Activation of autophagy has been shown in both the CRYAB p.Arg120Gly mouse model of desminopathy and muscle cell lines from patients with inclusion body myositis $(56,57)$. Macroautophagy is considered protective in late-onset neurodegenerative disorders such as Huntington disease and other polyglutamine expansion diseases, and it has been suggested that autophagy upregulation may be a tractable strategy for the treatment of a wide range of disorders (58). A similar approach should be considered in future development of treatments for some patients with desminopathy.

There are other cellular effects of myopathy-causing mutations in the genes encoding various cytoskeletal and sarcomeric proteins. Due to space constraints we are unable to discus these in detail, but many are explained in a recent review (59).

\section{Phenotypic features}

Desminopathy manifests with various phenotypes. Phenotypic variability depends on the type of inheritance and the location of mutations within the relatively large and structurally and functionally complex desmin molecule (60). The phenotype can further be influenced by interactions between desmin and other IF proteins and by the activity of chaperones and protein clearance mechanisms that may be capable of compensating for the detrimental effects of mutant desmin.

Clinical features of almost 200 reported desminopathy patients have been characterized as severe childhood-onset cardioskeletal

\section{Figure 4}

Myopathological images of skeletal muscle in patients with desminopathy. (A) Cross-section of a muscle biopsy from a patient with desminopathy, stained with trichrome and examined by light microscopy, shows characteristic amorphous deposits. (B) Serial sections stained with trichrome and immunostained for desmin indicate that these deposits contained desmin. (C) EM analysis of the same sample shows destruction of the $Z$ discs, which eventually leads to disorganization of myofibrils (see text for details). $\mathbf{B}$ and $\mathbf{C}$ adapted with permission from New England Journal of Medicine (61).

myopathy (4), adult-onset skeletal myopathy with cardiac involvement (13), pure skeletal myopathy $(61,62)$, cardiomyopathy with distal weakness $(15,63,64)$, pure dilated cardiomyopathy (65), and distal myopathy with cardiac, respiratory, bulbar, facial, and smooth muscle involvement $(5,66)$. Distal, limb-girdle phenotypes and scapuloperoneal weakness with variable cardiac and respiratory involvement have been seen within a set of kindreds segregating with the p.Arg350Pro desmin mutation (67).

Skeletal muscle disease. Progressive skeletal myopathy is one of the clinical variants of desminopathy (68) observed in almost a quarter of patients (69). The illness starts at a later age than in individuals with other disease variants, and the progression is very slow. Skeletal muscle weakness typically starts in distal leg muscles and spreads to proximal muscles, a trend that is about 3 times more common than the opposite (69). In developed disease, all four limbs are affected. Weakness in the trunk, neck, and facial muscles can also be seen in some patients (61). Muscle atrophy is a frequent observation. Electromyographic studies show motor unit potentials and abnormal electric irritability characteristic of myopathy (70).

Muscle imaging studies performed in patients with desminopathy have identified a recognizable pattern of muscle involvement (71). In the lower leg, the peroneal muscles display substantially more myopathic changes than either the tibialis anterior or the muscles of the posterior compartment (the soleus and the medial and lateral gastrocnemius). In the thigh, the earliest and always most severely affected muscle is the semitendinosus (a muscle at the back of the thigh), followed by the sartorius and gracilis. The effects on these muscles always exceeds the involvement of the adductor magnus, biceps femoris, and semimembranosus (71). The anterior compartment (the rectus femoris, vastus lateralis, intermedius, and medialis) is relatively spared. The gluteus maximus is more severely involved than gluteus medius and minimus. Generally, the severity of degeneration in distal muscles always exceeds that of proximal muscles (71).

Skeletal muscle biopsies of patients with desminopathy typically show irregularly shaped abnormal regions containing amorphous deposits that are best identified with modified trichrome stain as dark green or bluish material localized in the subsarcolemma or within the cytoplasm $(2,61,70)$ (Figure 4A). It requires a high degree of awareness to recognize these deposits, as some myopathologists consider them nonspecific. In addition, hyaline structures, cytoplasmic bodies, rods, and spheroid-like bodies have been reported $(13,61)$. Although not specific to desminopathy, rimmed vacuoles have been observed in most cases. Other nonspecific myopathic features, such as variation in fiber size, scattered atrophic fibers, and an increased number of internal nuclei are also frequently observed. Within the abnormal aggregates, proteins of diverse origins, including sarcomeric, cytoskeletal, and enzymatic proteins as well as proteins not known to be normally expressed 
in skeletal muscle, such as $A \beta$-amyloid and the amyloid precursor protein, have been identified $(72,73)$. The proteins most consistently present are desmin, $\alpha \mathrm{B}$-crystallin, dystrophin, myotilin, and ubiquitin $(2,15,61,66,73,74)$. The amount of desmin in affected muscle fibers of patients with desminopathy is increased, when compared with the amount in muscle from healthy individuals, as assessed by immunoblotting (75).

All desminopathy skeletal muscle specimens analyzed by EM have shown accumulation of granulofilamentous material denser than the $\mathrm{Z}$ disc and located beneath the sarcolemma and between the myofibrils (76) (Figure 4B). Characteristic $Z$ disc deformities, compact bodies of spheroid or cytoplasmic type, autophagic vacuoles containing myelin-like lamellae and debris (73), and focal groupings of mitochondria have also been observed $(15,26,30)$. In some cases, mitochondria are located alongside the granulofilamentous deposits, forming characteristic sandwich-like structures (76).

In the family in which disease segregates with the CRYAB p.Arg120Gly mutation, skeletal muscle biopsy has shown disorganization of filamentous networks and regions characteristic of desminopathy in which the intermyofibrillar network completely disappeared (77). Affected areas contain abnormal aggregates immunopositive for desmin, $\alpha \mathrm{B}$-crystallin, dystrophin, and ubiquitin. A subsarcolemmal and intermyofibrillar accumulation of dense granulofilamentous material with various degenerative changes was also observed by $\operatorname{EM}(6,77)$.

Respiratory dysfunction. Respiratory insufficiency can be a major cause of disability and death in desminopathy patients. It causes nocturnal hypoventilation with oxygen desaturation and, eventually, daytime respiratory failure (78). The diaphragm, unlike other skeletal muscles, functions in an environment in which forces can be transmitted in both longitudinal and transverse directions during each respiratory cycle, and desmin is the only molecule known to have dual orientation and serve as a viscoelastic element that dissipates mechanical energy in both planes (79). Progressive skeletal myopathy with early respiratory muscle involvement requiring respiratory support is observed in about a third of patients with desminopathy (69). A gradient between vital capacity in the upright and supine position of more than $30 \%$ suggests a preferential diaphragmatic involvement (78).

Cardiomyopathy. Atrioventricular conduction abnormalities requiring urgent implantation of a permanent pacemaker are a frequent feature of desminopathy attributed to the fact that the heart conduction system is rich in desmin (7). In a quarter of patients, the illness presents with cardiomyopathy (69), and cardiomyopathy alone with no skeletal muscle involvement has also been reported $(26,65,80,81)$. Heart disease is seen in more than $60 \%$ of patients in the advanced stages of illness (69). Some patients develop tachyarrhythmia requiring implantation of a cardioverter defibrillator (82). The age of disease onset in cases presenting with cardiomyopathy tends to be earlier and the illness more severe. Desminopathy-associated atrioventricular conduction blocks can be associated with dilated, restrictive, or hypertrophic cardiomyopathy $(26,75,80)$.

Variable pathogenic potentials of desmin mutations. One possible explanation for different desmin mutations causing either skeletal or cardiac myopathy is the type and location of the mutation. Mutations located in the N-terminal part of the $2 \mathrm{~B}$ helical segment of desmin cause primarily late onset skeletal muscle disease, with prolonged duration of illness and cardiomyopathy seen in only $36 \%$ of patients, whereas $60 \%-80 \%$ of patients with mutations in either the $1 \mathrm{~B}$ helical segment or the tail domain develop a more ominous disease with cardiac involvement (83). The earliest onset and the highest frequency of cardiomyopathy are associated with mutations located in the YRKLLEGEESRI motif in the C-terminal end of the 2B helical segment (frequency of cardiomyopathy $88 \%$ ). The disease is especially severe in carriers of the C-terminal p.Asp399Tyr, p.Glu401Lys, and p.Arg406Trp desmin mutations: age of onset is in the teens or early twenties, and life-threatening dysphagia, cardiomyopathy, and respiratory weakness develop within a few years $(23,24)$.

\section{Inheritance}

Autosomal dominant mutations. Eighty percent of desminopathy families show a classic autosomal dominant pattern of inheritance, most with full penetrance $(60,69)$. As some mutations are less pathogenic than others (23), in some autosomal dominant families, older members carrying the familial pathogenic mutation were found to be unaffected. The phenomenon of incomplete penetrance was first demonstrated in families with the p.Ile451Met desmin mutation $(65,68)$. In families with the p.Arg350Pro mutation, affected men had a higher risk of sudden cardiac death, suggesting a role for factors not immediately dependent on the location or type of mutation (67). In several thoroughly studied families, both parents were available for genetic testing and evidence was obtained that one of the parents was germ-line mosaic $(13,24)$. False paternity in these families was unequivocally excluded by haplotype analysis. Fourteen percent of desmin mutations occur de novo (69), suggesting that DES, in particular the part of the gene encoding the $2 \mathrm{~B}$ helical segment, is a hot spot for mutations.

Autosomal recessive mutations. In $6 \%$ of patients, the inheritance pattern has been determined to be autosomal recessive. Patients with recessive mutations develop the disease in infancy or childhood (at around 5 years of age) $(60,69)$. A homozygous deletion of 21 nucleotides that predicts an in-frame loss of seven amino acids from Arg173 through Glu179 (p.Arg173_Glu179del) in the 1B helical segment of desmin causes a severe clinical syndrome and compromises the ability of desmin to assemble into IFs in cell culture $(5,66)$. Even more devastating is the effect exerted by compound heterozygosity of p.Ala360Pro and p.Asn393Ile desmin mutations (4). A combination of p.Ala360Pro with p.Asn393Ile resulted in childhood onset of highly aggressive cardioskeletal myopathy, while neither of these two mutations was pathogenic in several surviving family members when they occurred in a heterozygous state (4). This observation fully correlates with the results of functional analysis in cultured cells: when expressed separately, p.Ala360Pro and p.Asn393Ile desmin mutants support the existing IF network in $\mathrm{C} 2 \mathrm{C} 12$ cells, but they are unable to do so when cotransfected (23).

Modifier genes. A patient with a combination of lamin A/C p.Arg644Cys and desmin p.Val469Met mutations developed severe muscle weakness and complete heart block, requiring heart transplantation (84). Immunohistochemistry of the explanted heart and biopsied skeletal muscle showed desmin aggregates and granulofilamentous electron dense material on EM, typical features of desminopathy. Desmin and lamin A/C proteins are believed to be indirectly connected via other IF proteins (84). Five other patients under our care have shown that a combination of mutations in DES and mutations or functional polymorphisms in other neuromuscular genes such as $\alpha$-glucosidase, ZASP, and MYOT substantially influences the disease phenotype (our unpublished observations). 


\section{Health care implications}

Diagnosis of desminopathy. Recognition of desminopathy requires careful correlation of clinical symptoms and findings from muscle histology and immunopathology. The disease may be clinically heterogeneous and the pattern of inheritance variable. The following criteria are important in arriving at the correct diagnosis $(2,35,61)$ : history of slowly progressive muscle weakness, dyspnea, and cardiac arrhythmias; demonstration of proximal and distal muscle weakness most often starting in the lower limbs and variably progressing to the upper extremities, trunk, neck flexors, and pharyngeal and facial muscles; restrictive ventilatory defect; EKG or cardiac monitoring showing conduction blocks or arrhythmias; EMG revealing myopathic units and abnormal spontaneous activity (fibrillation potentials, positive sharp waves, complex repetitive discharges, and occasional myotonic discharges); moderate elevation of the serum creatine kinase level; and characteristic alterations in frozen sections of muscle biopsy specimens highlighted by accumulation of amorphous or granular deposits that can be visualized with trichrome and H\&E stains, vacuoles, and chronic myopathic changes without inflammation. These accumulations represent ectopic deposits of myofibrillar proteins and should be immunopositive for desmin and dystrophin. EM analysis should show accumulation of granulofilamentous material under the sarcolemma and within the myofibrils; autophagic vacuoles are observed in some cases (61). Genetic testing is essential for establishing an accurate diagnosis of desminopathy and is necessary for providing appropriate genetic counseling and timely prevention of cardiac arrhythmia and heart failure (60).

Therapentic considerations. There is no specific treatment for desminopathy, but some of the complications and premature death can be prevented $(2,61)$. Early detection of cardiac arrhythmias and conduction defects is essential, since implantation of a pacemaker can be lifesaving. Individuals with progressive or life-threatening cardiomyopathy are candidates for cardiac transplantation. Respiratory support, consisting of continuous or bi-level positive airway pressure, initially at night and later at daytime, are indicated in patients with hypercapnea and other signs of incipient ventilatory failure. Risk of chest infection should be considered in these patients. Assistive devices should be used in individuals with advanced skeletal muscle weakness.

\section{Concluding remarks}

Desminopathy is associated with mutations in desmin and $\alpha \mathrm{B}-$ crystallin. Most of the known disease-associated desmin mutations affect amino acid residues that are crucial for filament assembly. They also render a dominant-negative effect on desmin function. In humans and transgenic mice, desmin mutants invoke accumulation of chimeric intracellular aggregates containing desmin and other cytoskeletal proteins. Desmin mutations show strikingly different pathogenic effects depending on their location, the type of amino acid change, the structural relationships these mutations disrupt, and the type of inheritance. $\alpha \mathrm{B}$-crystallin serves as a chaperone for desmin and, if mutated, can cause desminopathy. Current knowledge of the molecular pathogenesis of disorders resulting from desmin and $\alpha \mathrm{B}$-crystallin mutations is sufficient for some practical recommendations. However, there are multiple unresolved problems. We have limited understanding of the mechanisms involved in muscle-specific proteolytic systems and adaptive cellular pathways. Mechanisms leading to variable disease expression in skeletal or cardiac muscle cells also require additional studies. New technologies will help solve these problems and develop novel specific therapies.

\section{Acknowledgments}

This research was supported in part by the Intramural Research Program of the National Institute of Neurological Disorders and Stroke, NIH.

Address correspondence to: Lev G. Goldfarb, NIH, 5625 Fishers Lane, Room 4S06, Bethesda, Maryland 20892-9404, USA. Phone: (301) 402-1480; Fax: (301) 496-6341; E-mail: goldfarbl@ninds. nih.gov. Or to: Marinos C. Dalakas, Imperial College London, Hammersmith Hospital Campus, Du Cane Road, London W12 ONN, United Kingdom. Phone: 20-7594-7014; Fax: 20-7594-6548; E-mail: m.dalakas@imperial.ac.uk.
1. Goebel, H.H., et al. 1994. Immunohistologic and electron microscopic abnormalities of desmin and dystrophin in familial cardiomyopathy and myopathy. Rev. Neurol. (Paris). 150:452-459.

2. Selcen, D., and Engel, A.G. 2008. Myofibrillar myopathy. GeneReviews. http://www.ncbi.nlm.nih. gov/bookshelf/br.fcgi? book=gene\&part $=\mathrm{mfm}$.

3. Selcen, D., et al. 2008. Mutation in BAG3 causes severe dominant childhood muscular dystrophy. Ann. Neurol. 65:83-89.

4. Goldfarb, L.G., et al. 1998. Missense mutations in desmin associated with familial cardiac and skeletal myopathy. Nat. Genet. 19:402-403.

5. Muñoz-Mármol, A.M., et al. 1998. A dysfunctional desmin mutation in a patient with severe generalized myopathy. Proc. Natl. Acad. Sci. U. S. A. 95:11312-11317.

6. Vicart, P., et al. 1998. A missense mutation in the alpha-B-crystallin chaperone gene causes a desminrelated myopathy. Nat. Genet. 20:92-95.

7. Price, M.G. 1984. Molecular analysis of intermediate filament cytoskeleton - a putative load-bearing structure. Am. J. Physiol. 246:H566-H572.

8. Perng, M.D., et al. 1999. Intermediate filament interactions can be altered by HSP27 and alphaBcrystallin. J. Cell. Sci. 112:2099-2112.

9. Viegas-Péquignot, E., et al. 1989. Assignment of human desmin gene to band $2 \mathrm{q} 35$ by non-radioactive in situ hybridization. Hum. Genet. 83:33-36.
10. Li, Z., Lilienbaum, A., Butler-Browne, G., and Paulin, D. 1989. Human desmin-coding gene: complete nucleotide sequence, characterization and regulation of expression during myogenesis and development. Gene. 78:243-254.

11. Weber, K., and Geisler, N. 1985. Intermediate filaments: structural conservation and divergence. Ann. N. Y. Acad. Sci. 455:126-143.

12. Fuchs, E., and Weber, K. 1994. Intermediate filaments: structure, dynamics, function, and disease. Annu. Rev. Biochem. 63:345-382.

13. Park, K.Y., et al. 2000. Desmin splice variants causing cardiac and skeletal myopathy. J. Med. Genet. 37:851-857.

14. Conover, G.M., Henderson, S.N., and Gregorio, C.C. 2009. A myopathy-linked desmin mutation perturbs striated muscle actin filament architecture. Mol. Biol. Cell. 20:834-845.

15. Schröder, R., et al. 2003. On noxious desmin: functional effects of a novel heterozygous desmin insertion mutation on the extrasarcomeric desmin cytoskeleton and mitochondria. Hum. Mol. Genet. 12:657-669.

16. MacArthur, M.W., and Thornton, J.M. 1991. Influence of proline residues on protein conformation. J. Mol. Biol. 218:397-412.

17. Raats, J.M.H., et al. 1991. Assembly of carboxy-terminally deleted desmin in vimentin-free cells. Eur. J. Cell Biol. 56:84-103.
18. Brown, J.H., Cohen, C., and Parry, D.A.D. 1996. Heptad breaks in $\alpha$-helical coiled coils: stutters and stammers. Proteins. 26:134-145.

19. Strelkov, S.V., et al. 2002. Conserved segments $1 \mathrm{~A}$ and $2 \mathrm{~B}$ of the intermediate filament dimer: their atomic structures and role in filament assembly. EMBO J. 21:1255-1266

20. Strelkov, S.V., and Burkhard, P. 2002. Analysis of $\alpha$-helical coiled coils with the program TWISTER reveals a structural mechanism for stutter compensation. J. Struct. Biol. 137:54-64.

21. Kaminska, A., et al. 2004. Small deletions disturb desmin architecture leading to breakdown of muscle cells and development of skeletal or cardioskeletal myopathy. Hum. Genet. 114:306-313.

22. Herrmann, H., et al. 2000. The intermediate filament protein consensus motif of helix 2B: its atomic structure and contribution to assembly. J. Mol. Biol. 298:817-832.

23. Goudeau, B., et al. 2006. Variable pathogenic potentials of mutations located in the desmin alpha-helical domain. Hum. Mutat. 27:906-913.

24. Dagvadorj, A., et al. 2004. West European cluster of patients with severe cardioskeletal myopathy associated with a de novo R406W mutation in desmin. J. Neurol. 251:143-149.

25. Bar, H., et al. 2004. The biology of desmin filaments: how do mutations affect their structure, assembly, and organization. J. Struct. Biol. 148:137-152. 
26. Prusczyk, P., et al. 2006. Restrictive cardiomyopathy with atrioventricular conduction block resulting from a desmin mutation. Int. J. Cardiol. 117:244-253.

27. Herrmann, H., et al. 1996. Structure and assembly properties of the intermediate filament protein vimentin: the role of its head, rod and tail domains. J. Mol. Biol. 264:933-953.

28. Heimburg, T., Schuenemann, J., Weber, K., and Geisler, N. 1996. Specific recognition of coiled coils by infrared spectroscopy: analysis of the three structural domains of type III intermediate filament proteins. Biochemistry. 35:1375-1382.

29. Rogers, K.R., et al. 1995. Truncation mutagenesis of the non-alpha-helical carboxyterminal tail domain of vimentin reveals contributions to cellular localization but not to filament assembly. Eur. J. Cell Biol. 66:136-150.

30. Bar, H., et al. 2007. Conspicuous involvement of desmin tail mutations in diverse cardiac and skeletal myopathies. Hum. Mutat. 28:374-386.

31. Brakenhoff, R.H., et al. 1990. Human alpha B-crystallin (CRYA2) gene mapped to chromosome 11q12-q23. Hum. Genet. 85:237-240.

32. Dubin, R.A., Ally, A.H., Chung, S., and Piatigorsky, J. 1990. Human alpha-B-crystallin gene and preferential promoter function in lens. Genomics. 7:594-601.

33. Horwitz, J. 2000. The function of alpha-crystallin in vision. Semin. Cell Dev. Biol. 11:53-60.

34. Golenhofen, N., et al. 1999. Binding of the stress protein alpha B-crystallin to cardiac myofibrils correlates with the degree of myocardial damage during ischemia/reperfusion in vivo. J. Mol. Cell. Cardiol. 31:569-580.

35. Fardeau, M., et al. 1978. Une nouvelle affection musculaire familiale, definie par l'accumulation intra-sarco-plasmique d'un materiel granulo-filamentaire dense en microscopie electronique. Rev Neurol. 134:411-425.

36. Bova, M.P., et al. 1999. Mutation R120G in $\alpha$ B-crystallin, which is linked to a desmin-related myopathy, results in an irregular structure and defective chaperone-like function. Proc. Natl. Acad. Sci. U. S. A. 96:6137-6142.

37. Chávez Zobel, A.T., et al. 2003. Distinct chaperone mechanisms can delay the formation of aggresomes by the myopathy-causing R120G alphaB-crystallin mutant. Hum. Mol. Genet. 12:1609-1620.

38. Selcen, D., and Engel, A.G. 2003. Myofibrillar myopathy caused by novel dominant negative alpha-Bcrystallin mutations. Ann. Neurol. 54:804-810.

39. Li, Z., et al. 1996. Cardiovascular lesions and skeletal myopathy in mice lacking desmin. Dev. Biol. 175:362-366.

40. Milner, D.J., et al. 1996. Disruption of muscle architecture and myocardial degeneration in mice lacking desmin. J. Cell Biol. 134:1255-1270.

41. Capetanaki, Y., Milner, D.J., and Weitzer, G. 1997. Desmin in muscle formation and maintenance: knockouts and consequences. Cell Struct. Funct. 22:103-116.

42. Thornell, L., Carlsson, L., Mericskay, M., and Paulin, D. 1997. Null mutation in the desmin gene gives rise to a cardiomyopathy. J. Mol. Cell. Cardiol. 29:2107-2124.

43. Wang, X., et al. 2001. Mouse model of desmin-related cardiomyopathy. Circulation. 103:2402-2407.

44. Kostareva, A., et al. 2008. Mice expressing L345P mutant desmin exhibit morphological and functional changes of skeletal and cardiac mitochondria. J. Muscle. Res. Cell Motil. 29:25-36.
45. Wang, X., et al. 2002. Expression of R120G-aBcrystallin causes aberrant desmin and aB-crystallin aggregation and cardiomyopathy in mice. Circ. Res. 89:84-91.

46. Wang, X., Osinska, H., Gerdes, A.M., and Robbins, J. 2002. Desmin filaments and cardiac disease: establishing causality. J. Card. Fail. 8:S287-S292.

47. Perng, M.D., et al. 1999. The cardiomyopathy and lens cataract mutation in alpha B-crystallin alters its protein structure, chaperone activity and interaction with intermediate filaments in vitro. J. Biol. Chem. 274:33235-33243.

48. Bar, H., et al. 2005. Severe muscle disease-causing desmin mutations interfere with in vitro filament assembly at distinct stages. Proc. Natl. Acad. Sci.U.S. A. 102:15099-15104.

49. Bar, H., et al. 2006. Forced expression of desmin and desmin mutants in cultured cells: impact of myopathic missense mutations in the central coiled-coil domain on network formation. Exp. Cell Res. 312:1554-1565.

50. Bar, H., et al. 2006. Impact of disease mutations on the desmin filament assembly process. J. Mol. Biol. 360:1031-1042.

51. Li, M., and Dalakas, M.C. 2001. Abnormal desmin protein in myofibrillar myopathies caused by desmin gene mutations. Desmin protein in myofibrillar myopathies. Ann. Neurol. 49:532-536.

52. Liu, J., et al. 2006. Impairment of the ubiquitin proteasome system in desminopathy mouse hearts. FASEB J. 20:362-364.

53. Liu, J., Tang, M., Mestril, R., and Wang, X. 2006. Aberrant protein aggregation is essential for a mutant desmin to impair the proteolytic function of the ubiquitin-proteasome system in cardiomyocytes. J. Mol. Cell. Cardiol. 40:451-454.

54. Sanbe, A., et al. 2004. Desmin-related cardiomyopathy in transgenic mice: A cardiac amyloidosis. Proc. Natl. Acad. Sci. U. S. A. 101:10132-10136.

55. Dalakas, M.C. 2006. Sporadic inclusion body myositis: diagnosis, pathogenesis and therapeutic strategies. Nat. Clin. Pract. Neurol. 2:437-447.

56. Lünemann, J.D., et al. 2007. Beta-amyloid is a substrate of autophagy in sporadic inclusion body myositis. Ann. Neurol. 61:476-483.

57. Tannous, P., et al. 2008. Autophagy is an adaptive response in desmin-related cardiomyopathy. Proc. Natl. Acad. Sci. U. S. A. 105:9745-9750.

58. Sarkar, S., and Rubinsztein, D.C. 2008. Huntington's disease: degradation of mutant huntingtin by autophagy. FEBS J. 275:4263-4270.

59. Ferrer, I., and Olivé, M. 2008. Molecular pathology of myofibrillar myopathies. Expert Rev. Mol. Med. 10:e25.

60. Goldfarb, L.G., Vicart, P., Goebel, H.H., and Dalakas, M.C. 2004. Desmin myopathy. Brain. 127:723-734.

61. Dalakas, M.C., et al. 2000. Desmin myopathy, a skeletal myopathy with cardiomyopathy caused by mutations in the desmin gene. N. Engl. J. Med. 342:770-780.

62. Dalakas, M.C., et al. 2003. Progressive skeletal myopathy, a phenotypic variant of desmin myopathy associated with desmin mutations. Neuromuscul. Disord. 13:252-258.

63. Bertini, E., et al. 1991. Neuromyopathy and restrictive cardiomyopathy with accumulation of intermediate filaments: a clinical, morphological and biochemical study. Acta Neuropathol. 81:632-640.

64. Lobrinus, J.A., et al. 1998. Familial cardiomyopathy and distal myopathy with abnormal desmin accumulation and migration. Neuromuscul. Disord. 8:77-86.

65. Li, D., et al. 1999. Desmin mutation responsible for idiopathic dilated cardiomyopathy. Circulation. 100:461-464.

66. Ariza, A., et al. 1995. Desmin myopathy: a multisystem disorder involving skeletal, cardiac, and smooth muscle. Hum. Pathol. 26:1032-1037.

67. Walter, M.C., et al. 2007. Scapuloperoneal syndrome type Kaeser and a wide phenotypic spectrum of adult-onset, dominant myopathies are associated with the desmin mutation R350P. Brain. 130:1485-1496.

68. Dalakas, M.C., et al. 2002. Desmin myopathy: distinct filamentopathy caused by mutations in the desmin gene. Acta Myol. 21:138-143.

69. Goldfarb, L.G., Olivé, M., Vicart, P., and Goebel, H.H. 2008. Intermediate filament diseases: desminopathy. In The sarcomere and skeletal muscle disease. N.G. Laing, editor. Springer Science. New York, New York, USA. Landes Bioscience. Austin, Texas, USA. 131-164.

70. Selcen, D., Ohno, K., and Engel, A.G. 2004. Myofibrillar myopathy: clinical, morphological and genetic studies in 63 patients. Brain. 127:439-451.

71. Fischer, D., et al. 2008. Distinct muscle imaging patterns in myofibrillar myopathies. Neurology. 71:758-765.

72. Olivé, M., et al. 2004. Desmin-related myopathy: clinical, electrophysiological, radiological, neuropathological, and genetic studies. J. Neurol. Sci. 219:125-137.

73. Vrabie, A., et al. 2005. The enlarging spectrum of desminopathies: new morphological findings, eastward geographic spread, novel exon 3 desmin mutation. Acta Neuropathol. 109:411-417.

74. Olivé, M., et al. 2007. Phenotypic patterns of desminopathy associated with three novel mutations in the desmin gene. Neuromuscul. Disord. 17:443-450.

75. Bar, H., et al. 2005. Pathogenic effects of a novel heterozygous R350P desmin mutation on the assembly of desmin intermediate filaments in vivo and in vitro. Hum. Mol. Genet. 14:1251-1260.

76. Claeys, K.G., et al. 2008. Electron microscopy in myofibrillar myopathies reveal clues to the mutated gene. Neuromusc. Disord. 18:656-666.

77. Fardeau, M., et al. 2000. Myopathie familiale avec surcharge en desmine, sous forme de matériel granulo-filamentaire dense en microscopie électronique, avec mutation dans le gène de l'alpha-B-cristalline. Rev. Neurol. (Paris). 156:497-504.

78. Dagvadorj, A., et al. 2003. Respiratory insufficiency in desminopathy patients caused by introduction of proline residues in desmin C-terminal $\alpha$-helical segment. Muscle Nerve. 27:669-675.

79. Boriek, A.M., et al. 2001. Desmin integrates the three-dimensional mechanical properties of muscles. Am. J. Physiol. Cell Physiol. 280:C46-C52.

80. Arbustini, E., et al. 2006. Desmin accumulation restrictive cardiomyopathy and atrioventricular block associated with desmin gene defects. Eur. J. Heart Fail. 8:477-483.

81. Taylor, M.R.G., et al. 2007. Prevalence of desmin mutations in dilated cardiomyopathy. Circulation. 115:1244-1251.

82. Luethje, L.G., et al. 2004. Prophylactic implantable cardioverter defibrillator placement in a sporadic desmin related myopathy and cardiomyopathy. Pacing Clin. Electrophysiol. 27:559-560.

83. Kostera-Pruszczyk, A., et al. 2008. Diversity of cardiomyopathy phenotypes caused by mutations in desmin. Int. J. Cardiol. 131:146-147.

84. Muntoni, F., et al. 2006. Disease severity in dominant Emery Dreifuss is increased by mutations in both emerin and desmin proteins. Brain. 129:1260-1268. 